\title{
SOLUCIONES LOCALES Y GLOBALES EN UNA ECUACIÓN PARABÓLICA SEMILINEAL
}

\author{
Nancy Moya Lazáro*, Félix Pariona Vilca ${ }^{* *}$, Carlos Castañeda Yaya ${ }^{* * *}$ \\ Jacinto Mendoza Solís ${ }^{* * * *}$ \& Luis Núñez Ramirez ${ }^{* * * * *}$
}

\begin{abstract}
Resumen: En este trabajo presentamos el estudio de la existencia local, existencia global y la solución de un problema parabólico semilineal. El estudio lo hacemos específicamente, estudiando la ecuación del calor con condiciones de borde Dirichlet en un dominio abierto, acotado: de frontera regular. Abordamos el estudio de la solución local y global, presentamos estimaciones de la solución en espacios finos, que con una hipótesis sobre la no-linealidad, en principio con una condición de signo y luego una condición sobre la derivada, nos permite concluir que existe solución global del problema.
\end{abstract}

Palabras clave: Semigrupo, solución local, solución global, técnicas de comparación, monotonía, positividad.

\section{LOCAL AND GLOBAL SOLUTIONS IN A SEMILINEAR PARABOLIC EQUATION}

\begin{abstract}
In this paper we present the study of the local and global existence and the solution of a semilinear parabolic problem. The study has been specifically done through the equation of heat with Dirichlet boundary conditions in a regular - border, marked and open domain. We deal with the local and global solution, present the estimates of the solution in space years that under a hypothesis on non-linearity, first on a sign condition and then on derivative one, allows us to conclude the existence of a global solution of the problem.
\end{abstract}

Key words: Semigroup, local solution, comparison techniques, monotony, positivity.

\section{Introducción}

En este trabajo planteamos el estudio de la existencia local y global de solución de un problema parabólico semilineal. El problema que estudiamos es el siguiente

$$
\left\{\begin{array}{l}
\partial_{t} u-\Delta u=f(u), x \in \Omega \\
u(0)=u_{0} \\
\left.u\right|_{\Gamma=0}
\end{array}\right.
$$

donde. $\Omega \subset \mathbb{R}^{N}$ es un conjunto abierto acotado, de frontera regular $\Gamma=\partial \Omega$

$u_{0} \in H_{0}^{1}, f: \mathbb{R} \rightarrow \mathbb{R}$.

En principio, para obtener un resultado de existencia local de solución del problema (1) debemos imponer ciertas condiciones de crecimiento a la parte no lineal. Para el tratamiento de soluciones globales: dividimos nuestro estudio en: primero analizamos en una clase de no-linealidades que presenten condiciones de signo y luego en otra clase de no-linealidades que tienen la derivada acotada. superiormente. Encontramos estimaciones finas para la solución, y con condiciones naturales sobre la no-linealidad conseguimos la solución global.

\footnotetext{
*UNMSMS, Facultad de Ciencias Matemáticas, e-mail: nanesp@̈yahoo.com

***NMSMS, Facultad de Ciencias Matemáticas, e-mail: fparionav@hotmail.com

${ }^{* * *}$ UNMSMS, Facultad de Ciencias Matemáticas

**+* UNMSMSS. Facultad de Ciencias Matemáticas, e-mail: pedromendozas87 cihotmail.com

****** UMMSMIS, Facultad de Ciencias Matemáticas, e-mail: Inumeztaffarel27@yahoo.com.pe
} 


\section{El Semigrupo de $-\Delta_{D}$}

Nuestro marco funcional es el espacio base $X=L^{2}(\Omega)$. Entonces el operador $-\Delta_{D}$ Laplaciano con condiciones de contorno Dirichlet, genera un semigrupo analítico en $X$, que denotamos $e^{\Delta t}$. De acuerdo a [3], podemos definir una escala de espacios de interpolación, $X^{\alpha}$ para todo $\alpha \in \mathbb{R}$ de manera que $X^{0}=L^{2}(\Omega), \quad X^{\frac{1}{2}}=H_{0}^{1}(\Omega)$, y $X^{1}=\operatorname{Dom}(-\Delta)=H^{2}(\Omega) \cap H_{0}^{1}(\Omega)$.

Además tenemos las siguientes estimaciones del semigrupo entre los distintos espacios de la escala.

Teorema 2.1. Para todo $t \geq 0$ y $\alpha, \beta \in \mathbb{R}$,

$$
\left\|e^{\Delta t}\right\|_{\alpha, \beta} \leq M t^{-(\beta-\alpha)} e^{-\lambda_{1} t}
$$

donde $\lambda_{1}$ es el primer autovalor de $-\Delta y\left\|e^{\Delta t}\right\|_{\alpha, \beta}$ denota la norma del semigrupo actuando entre $X^{\alpha}$ y $X^{\beta}$.

Así mismo, tenemos las siguientes estimaciones del semigrupo actuando de $L^{p}(\Omega)$ en $L^{q}(\Omega)$

Lema 2.1. Sea $1 \leq p \leq q \leq \infty$. Entonces

$$
\left\|e^{\Delta t} u\right\|_{L^{q}(\Omega)} \leq C t^{-\frac{N}{2}\left(\frac{1}{p}-\frac{1}{q}\right)}\|u\|_{L^{p}(\Omega)}
$$

para todo $u \in L^{p}(\Omega)$ y $t>0$ donde $C=C(N, p, q)$.

El espacio $X^{\alpha}$, con $\alpha \geq 0$ puede dotarse de una estructura de espacio de Banach ordenado, ver[2] con el orden siguiente

$$
u \leq v \text { si } y \text { solo si } u(x) \leq v(x) \text { ctp } x \text {. }
$$

Este orden, verifica además

$$
x \geq 0 \text { si y solo si }\langle x, y\rangle \geq 0 \text { para todo } y \geq 0
$$

Utilizando esta propiedad podemos definir un orden en $X^{-\alpha}$, para $\alpha>0$ de la siguiente manera

$$
f \leq g \text { siy solo si }\langle f, \phi\rangle_{-\alpha, \alpha} \leq\langle g, \phi\rangle_{-\alpha, \alpha} \text { para todo } \phi \geq 0, \phi \in X^{\alpha}
$$

Una propiedad importante del semigrupo del calor es la siguiente

Teorema 2.2. El semigrupo del calor, $e^{\Delta t}$, es creciente y positivo. En particular conserva el orden y el signo, es decir, si $u_{0} \geq u_{1}$ entonces $e^{\Delta t} u_{0} \geq e^{\Delta t} u_{1}$, y si $u_{0} \geq 0$ entonces $e^{\Delta t} u_{0} \geq 0$.

Para más detalle véase[2]

\section{Existencia de solución local}

Sea $\Omega \subset \mathbb{R}^{N}$ un abierto acotado regular. Analizamos ahora la existencia local y regularidad de soluciones; más precisamente, estudiamos el siguiente problema

$$
\left\{\begin{array}{l}
\partial_{t} u-\Delta u=f(u) . c n \Omega \\
u(0)=u_{0} \\
\left.u\right|_{\Gamma}=0
\end{array}\right.
$$

donde $u_{0} \in H_{0}^{1}(\Omega)=X^{\frac{1}{2}}$ y $f: \mathbb{R} \rightarrow \mathbb{R}$.

Teorema 3.1. Consideramos el problema (5) con $u_{0} \in X^{\frac{1}{2}}=H_{0}^{1}(\Omega)$. Asumimos que la parte no lineal f satisface

$$
\left|f^{\prime}(s)\right| \leq C\left(1+|s|^{p-1}\right)
$$

$\operatorname{con} C \geq 0,1 \leq p<\frac{N+2}{N-2}$.

Entonces, existe una única solución local del problema (5). Ademús $u \in C\left([0, \tau)\right.$. $\left.H_{0}^{1}(\Omega)\right)$ dada por la fórmula de. variación de las constantes,

$$
u\left(t, u_{0}\right)=S(t) u_{0}+\int_{0}^{t} S(t-s) f_{c}(u(s)) d s, \text { para todo } 0 \leq t<\tau\left(u_{0}\right)
$$

donde $S^{\prime}(t)$ denota el semigrupo analítico generado por $\Delta$, esto es $S(t)=e^{\Delta t} y\left[0, \tau_{0}\right)$ es el intervalo maximal de. existencia. 
Demostración. De acuerdo al Teorema de existencia y unicidad, ver [3]. Primero, probaremos que el operador de Nemitcky de la función $f$, operador de composición, transforme $H_{0}^{1}(\Omega)$ en $X^{-\beta}$ con $0 \leq \beta<\frac{1}{2}$, también se probará que $f^{e}: H_{0}^{1}(\Omega) \rightarrow X^{-\beta}$ es lipschitz sobre acotados.

Observemos que la condición (6), implica que

$$
|f(s)| \leq C\left(1+|s|^{p}\right)
$$

para alguna constante $C$, de lo cuál se sigue que el operador de Nemitcky asociado a $f$ transforma $L^{q}(\Omega)$ en $L^{\frac{q}{p}}(\Omega)$ y entre estos espacios es acotado y continuo. También como $f$ satisface (6), el operador de Nemitcky asociado a $f$ es Lipschitz sobre acotados entre esos espacios.

Por otro lado, de las inclusiones continuas de Sobolev se tiene que $H_{0}^{1}(\Omega) \subset L^{2^{*}}(\Omega)$ con

$$
2^{*} \begin{cases}\leq \infty, & \text { si } N=1 \\ <\infty, & \text { si } N=2 \\ =\frac{2 N}{N-2}, & \text { si } N \geq 3\end{cases}
$$

Luego, si $u \in H_{0}^{1}(\Omega)$, por (8), $f(u) \in L^{\frac{2^{*}}{p}}(\Omega)$.

Ahora necesitamos que para algún $\beta \in\left[0, \frac{1}{2}\right)$ se realice el siguiente diagrama de inclusiones continuas

$$
H_{0}^{1}(\Omega) e, t f s, l X^{-\beta} L^{2^{*}(\Omega)} e, t f L^{\frac{2^{*}}{p}}(\Omega) n, r
$$

para ello, bastará $X^{\beta} \subset H^{2 \beta}(\Omega) \subset L^{\left(\frac{2^{*}}{p}\right)^{\prime}}(\Omega)$, es decir

$$
2 \beta-\frac{N}{2} \geq-\frac{N}{\left(\frac{2^{*}}{p}\right)^{\prime}}
$$

Existirá un $0 \leq \beta<\frac{1}{2}$ verificando (10) si y solo si

$$
1-\frac{N}{2}>-\frac{N}{\left(\frac{2^{*}}{p}\right)^{\prime}}
$$

es decir, si y solo si

$$
p<\frac{N+2}{N-2}=1+\frac{2.2}{N-2}
$$

Por tanto se cumplen las hipótesis del Teorema 3.3.3 de existencia y unicidad de [3].

En el caso que $N \leq 2$, tenemos la inyección $H_{0}^{1}(\Omega) \hookrightarrow L^{p}(\Omega)$ con $p$ arbitrario, $p<\propto$ y entonces (11) se verifica siempre. $\square$

Teorema 3.2. Con las condiciones del teorema anterior, existe una única solución maximal definida en $\left[0, T_{x}\right)$. Además, si $T_{\infty}<+\infty$, la solución explota en $T_{\infty}$, es decir

$$
\lim _{t \rightarrow T_{\varkappa}}\|u(t)\|_{H_{0}^{1}(\Omega)}=\infty
$$

Suponemos en adelante que $f$ verifica (6).

\section{Condiciones sobre el segundo miembro para tener explosión en tiempo finito}

Vamos a ver, que imponiendo ciertas condiciones sobre el segundo miembro, tenemos que existen datos iniciales para los cuales la solución del problema (5). explota en tiempo finito. Para ello, supongamos que se verifica la siguiente propiedad

$$
\text { Si } u_{0} \geq 0 \text {, entonces } u(t) \geq 0 \text { para todo } t \geq 0 \text {. }
$$

En la sección siguiente, veremos que para que se verifique (14) basta $f(0) \geq 0$.

Sea $\varphi$ la primera función propia del $-\Delta$, es decir, la solución del problema

$$
\left\{\begin{array}{l}
-\Delta u=\lambda_{1} \varphi, \text { en } \Omega \\
\left.\varphi\right|_{\tau}=0
\end{array}\right.
$$


donde $\lambda_{1}>0$ es el primer autovalor del $-\Delta$. Sabemos que $\varphi$ es positiva. Definimos ahora $z(t)$ como

$$
z(t)=\int_{\Omega} u(t) \varphi
$$

la proyección de la solución sobre la primera función propia de $-\Delta$.

Multiplicando la ecuación por $\varphi$ e integrando en $\Omega$, tenemos

$$
\int_{\Omega} u_{t} \varphi-\int_{\Omega} \Delta u \varphi=\int_{\Omega} f(u) \varphi
$$

de donde, integrando por partes dos veces en la segunda integral y teniendo en cuenta la ecuación que verifica $\varphi$,

$$
z^{\prime}(t)=-\lambda_{1} z(t)+\int_{\Omega} f(u) \varphi
$$

Si $f$ es convexa, eligiendo $\varphi$ de manera que $\int_{\Omega} \varphi=1$, estamos en condiciones de aplicar la desigualdad de Jensen obteniendo,

$$
z^{\prime}(t) \geq-\lambda_{1} z(t)+f(z(t))
$$

Llamando $h(z)=-\lambda_{1} z+f(z)$. Si $h(z)$ tiene un cero, $z^{*}$, a partir del cual es positiva, entonces si $z(0)=\int_{\Omega} u_{0} \varphi>z^{*}$. la solución $z(t)$. crece indefinidamente pero queremos que la solución explote en tiempo finito. Para ello: consideramos la ecuación

$$
z^{\prime}(t)=h(z(t))
$$

Integrando en tiempo,

$$
\int_{0}^{t} \frac{z^{\prime}(s)}{h(z(s))} d s=t
$$

luego

$$
\int_{\tilde{z}(0)}^{z(t)} \frac{1}{h(s)} d s=t
$$

Para tener explosión en tiempo finito. basta

$$
\int_{z(0)}^{\infty} \frac{1}{h(s)} d s=T<\infty
$$

y en este caso, la solución explota en un tiempo $0<T_{0}<T$.

Teorema 4.1. Si $f$ es convexa, es tal que se verifica (14) y

$$
\int^{x} \frac{1}{f(t)-\lambda_{1} t} d t<\infty
$$

con $\lambda_{1}$ el primer autovalor de $-\Delta$. entonces si $u_{0}$ es tal que

$$
\int_{\Omega} u_{0 \varphi} \geq z^{*}
$$

la solución del problema explota en ticmpo finito.

Por $\int^{x}$ denotamos la integral sobre un intervalo de la forma $\left\langle t_{1},+\infty\right\rangle$. para $\left.t_{1}\right\rangle t_{0}$ para cierto $t_{0}$.

\section{Resultados de Monotonía}

Dotamos al espacio $X^{\alpha}$ con una estructura de espacio de Banach ordenado ver [2] con el orden siguiente.

$$
u \leq v \text { si y solo si } u(x) \leq v(x) \text { ctp } x \text {. }
$$

Teorema 5.1. Si $f: \mathbb{R} \rightarrow \mathbb{R}$ es localmente Lipschitz, $f(0) \geq 0$ y $u_{0} \geq 0$. entonces $u(t) \geq 0$ para todo $t \geq 0$. 
Teorema 5.2. Si $u\left(t, u_{i}\right)$ con $i=1,2$ es la solución del problema

$$
\left\{\begin{array}{l}
u_{t}-\Delta u+\lambda u=f_{i}(u) \\
u_{0}=u_{i} \\
\left.u\right|_{\Gamma}=0
\end{array}\right.
$$

con $u_{1} \leq u_{2}, f_{1}(.) \leq f_{2}($.$) , entonces u\left(t, u_{1}\right) \leq u\left(t, u_{2}\right)$ para todo $t$ donde la solución exista.

Los dos teoremas anteriores se demuestran fácilmente a partir de la fórmula de variación de las constantes, para la solución del problema (1) en el caso en que, o bien $f$ sea creciente (o positiva) o exista una constante $\beta \in \mathbb{R}$ tal que $f+\beta I$ sea creciente (o positiva), o bien las soluciones sean acotadas. En este último caso, podemos encontrar $\beta \in \mathbb{R}$ tal que $f+\beta I$ sea creciente (o positiva). Esta condición en el caso de ser creciente, se lee como $f^{\prime}(s)>-\beta$ para todo $s \in \mathbb{R}$, es decir pedimos una acotación inferior de crecimiento de $f$. En el caso de ser positiva, la condición se lee como $f(s) \geq-\beta s$ para todo $s \in \mathbb{R}$, es decir, pedimos que la función $f$ esté por encima de una recta.

\section{Existencia global de solución}

Veamos ahora, condiciones sobre el segundo miembro que aseguren la existencia global de solución del problema (5).

\subsection{Condición de estructura o de signo.}

Supongamos que $f$ verifica la siguiente condición de estructura

$$
s f(s) \leq-C_{0}|s|^{2}+C_{1}|s| \text {, para todo } s \in \mathbb{R}
$$

donde $C_{1} \geq 0$.

La hipótesis (28) implica, en particular que para $s \geq 0$,

$$
f(s) \leq-C_{0} s+C_{1} .
$$

Estamos pidiendo así, que $f$ tenga un crecimiento sublineal en los puntos donde $s \geq 0$, es decir estamos pidiendo que la función $f$ no sea demasiado positiva. Si $f$ tuviese un crecimiento superlineal, tendríamos una función convexa por encima de una recta que esta por debajo de $f$.

La condición (28) es equivalente a

$$
\limsup _{|s| \rightarrow x} \frac{f(s)}{s} \leq-C_{0}^{\prime}
$$

para $C_{0}^{\prime}<C_{0}$ que es una condición sobre el crecimiento de $f$ en el infinito. Esto se tiene en particular si $f$ verifica

$$
\limsup _{|s| \rightarrow \infty} f^{\prime}(s) \leq-C_{0}^{\prime}
$$

Teorema 6.1. Supongamos que f verifica la siguiente condición de signo

$$
s f(s) \leq-C_{0}|s|^{2}+C_{1}|s|, \text { para todo } s \in \mathbb{R}
$$

donde $C_{1} \geq 0$. Entonces toda solución del problema (5) con condición inicial $u_{0} \in H_{0}^{1}(\Omega)$ está globalmente definida en $H_{0}^{1}(\Omega)$.

Demostración. La prueba la haremos de la siguiente manera, de la condición de (30) establecemos un problema lineal con solución $w\left(t,\left|u_{0}\right|\right)$ globalmente definida. Mediante técnicas de comparación se prueba la siguiente relación $\left|u\left(t, u_{0}\right)\right| \leq w\left(t,\left|u_{0}\right|\right)$. Así obtenemos estimaciones buenas de $w\left(t,\left|u_{0}\right|\right)$ en los espacios $H^{2 \alpha, q}(\Omega)$, con $\alpha \in[0,1)$, utilizando la fórmula de variación de las constantes se obtienen estimaciones de $u(t)$.

En efecto,

(1) Tenemos que $\Delta-C_{0} I$ es el generador infinitesimal de un semigrupo analítico, que preserva orden en $X^{\beta}$ : para $0<\beta<1$ y mantiene invariante la escala de espacios del $-\Delta$. 
(2) Una vez visto esto y con $C_{1} \geq 0$ el problema lineal

$$
\left\{\begin{array}{l}
w_{t}-\Delta w=-C_{0} w+C_{1} \\
w(0)=\left|u_{0}\right| \in H_{0}^{1}(\Omega)=X^{\frac{1}{2}} \\
\left.w\right|_{\Gamma}=0
\end{array}\right.
$$

está bien definido, lo que implica que existe una única solución denotada por $w^{+}\left(t,\left|u_{0}\right|\right)$ definida para todo $t \geq 0$, que está explícitamente dada por la fórmula de variación de las constantes.

$$
w^{+}\left(t,\left|u_{0}\right|\right)=e^{\left(\Delta-C_{0}\right) t}\left|u_{0}\right|+\int_{0}^{t} e^{\left(\Delta-C_{0}\right)(t-s)} C_{1} d s
$$

(3) Como $\Delta-C_{0} I$ es el generador infinitesimal de un semigrupo analítico, que denotamos por $e^{\left(\Delta-C_{0} I\right) t}$, que preserva orden y como $C_{1} \geq 0, w(0)=\left|u_{0}\right| \geq 0$ entonces

$$
w^{+}\left(t,\left|u_{0}\right|\right) \geq 0, \text { para todo } t>0 .
$$

(4) Por otra parte de la condición de signo (28), tenemos que

$$
f(s) \leq-C_{0} s+C_{1}, \text { para } s \geq 0
$$

y como $u_{0} \leq\left|u_{0}\right|$, entonces por las técnicas de comparación seguimos que

$$
u\left(t, u_{0}\right) \leq w^{+}\left(t,\left|u_{0}\right|\right)
$$

Procediendo similarmente, consideramos el problema auxiliar donde $w^{-}\left(t,-\left|u_{0}\right|\right)$ es solución de

$$
\left\{\begin{array}{l}
w_{t}-\Delta w=-C_{0} w-C_{1} \\
w(0)=-\left|u_{0}\right| \in H_{0}^{1}(\Omega)=X^{\frac{1}{2}} \\
\left.w\right|_{\Gamma}=0
\end{array}\right.
$$

De la condición de signo (28) tenemos también que

$$
f(s) \geq-C_{0}(s)-C_{1} \text {, para todo } s<0
$$

y como $u_{0} \geq-\left|u_{0}\right|$ entonces por las técnicas de comparación seguimos que

$$
-w^{+}\left(t,\left|u_{0}\right|\right):=w^{-}\left(t,-\left|u_{0}\right|\right) \leq u\left(t, u_{0}\right)
$$

lo cuál implica

$$
-u\left(t, u_{0}\right) \leq u^{+}\left(t,\left|u_{0}\right|\right)
$$

Por (34) y (37)

$$
\left|u\left(t, u_{0}\right)\right| \leq w^{+}\left(t,\left|u_{0}\right|\right)
$$

para todo $t>0 \cdot \square$

La relación anterior nos dice que las estimaciones de $w^{+}\left(t,\left|u_{0}\right|\right)$ son también estimaciones para $\left|u\left(t, u_{0}\right)\right|$. A continuación. veamos si la globalidad de $u^{+}\left(t,\left|u_{0}\right|\right)$ le da la globalidad a la solución $u\left(t, u_{0}\right)$.

Lema 6.1. Existe una constante $C_{T}>0$ tal que, para todo $0<t_{0}<t<T$ se verifica

$$
\|u(t)\|_{L^{\infty}(\Omega)}<C_{T}
$$

Demostración. Por (38), basta probar que $w^{+}\left(t,\left|u_{0}\right|\right)$ está acotada en $L^{\infty}(\Omega)$ en intervalos de tiempos finitos. con lo que tendremos una estimación $L^{\infty}(\Omega)$ para $u\left(t, u_{0}\right)$. Para ello vamos a usar el Lema 2.1, que nos da estimaciones del semigrupo generado por $\Delta$ actuando de $L^{p}(\Omega)$ en $L^{q}(\Omega)$.

Sea $g(t)=C_{1} \in L^{\infty}(\Omega) \subset L^{2}(\Omega)$. Por lia fórmula de variación de las constantes para $w^{+}\left(t,\left|u_{0}\right|\right)$ tenemos

$$
w^{+}\left(t,\left|u_{0}\right|\right)=e^{\left(\Delta-C_{0}\right) t}\left|u_{0}\right|+\int_{0}^{t} e^{\left(\Delta-C_{0}\right)(t-s)} C_{1} d s
$$


luego

$$
\begin{aligned}
\left\|w^{+}\left(t,\left|u_{0}\right|\right)\right\|_{L^{\infty}(\Omega)} & \leq\left\|e^{\left(\Delta-C_{0}\right) t}\left|u_{0}\right|\right\|_{L^{\infty}(\Omega)} \\
& +\int_{0}^{t}\left\|e^{\left(\Delta-C_{0}\right)(t-s)} C_{1}\right\|_{L^{\infty}(\Omega)} d s
\end{aligned}
$$

Ahora bien, por un lado, usando el Lema 2.1

$$
\left\|e^{\left(\Delta-C_{0}\right) t}\left|u_{0}\right|\right\|_{L^{\infty}(\Omega)} \leq C e^{D t} t^{\frac{-N}{4}}\left\|u_{0}\right\|_{L^{2}(\Omega)} \leq C_{T}\left\|u_{0}\right\|_{H_{0}^{1}(\Omega)} \leq C_{T}
$$

para $t_{0} \leq t \leq T$ y cierto $D \in \mathbb{R}$.

Observamos que, como estamos en intervalos acotados de tiempo, para toda constante $D$, tenemos una cota para $e^{D t}$ uniforme en $\left[t_{0}, T\right]$ por lo que no importa el signo de dicha constante, D.

Por otro lado, volviendo a usar el Lema 2.1 , para todo $t_{0} \leq t \leq T, D \in \mathbb{R}$ conseguimos

$$
\left\|e^{\left(\Delta-C_{0}\right)(t-s)} C_{1}\right\|_{L^{\infty}(\Omega)} \leq C e^{D(t-s)}
$$

luego,

$$
\int_{0}^{t}\left\|e^{\left(\Delta-C_{0}\right)(t-s)} C_{1}\right\|_{L^{\infty}(\Omega)} d s \leq \int_{0}^{t} C e^{D(t-s)} d s \leq C_{T}
$$

Por tanto,

$$
\left\|w^{+}\left(t,\left|u_{0}\right|\right)\right\|_{L^{\infty}(\Omega)} \leq C_{T}
$$

y por $(38)$

$$
\|u(t)\|_{L^{\infty}(\Omega)} \leq C_{T}, \text { para todo } t \in\left[t_{0}, T\right] \cdot \square
$$

A continuación gracias a la fórmula de variación de las constantes, obtenemos estimaciones en espacios más finos para la solución.

Lema 6.2. Existe una constante $C_{T}>0$ tal que para todo $0<t_{0}<t<T$ se verifica

$$
\|u(t)\|_{H_{0}^{\prime}(?)}<C_{T}
$$

Demostración. Por el lema anterior tenemos que para $0<t_{0} \leq t \leq T$.

$$
\|f(u(t))\|_{L^{\infty}(\Omega)}<C_{T}
$$

De la fórmula de variación de las constantes.

$$
u(t)=e^{\Delta\left(t-t_{0}\right)} u\left(t_{0}\right)+\int_{t_{0}}^{t} e^{\Delta(t-s)} f(u(s)) d s
$$

luego

$$
\begin{aligned}
\|u(t)\|_{H_{0}^{\prime}(\Omega)} & \leq\left\|e^{\Delta\left(t-t_{0}\right)}\right\|_{\frac{1}{2} \cdot \frac{1}{2}}\left\|u\left(t_{0}\right)\right\|_{\left.H_{0}^{\prime}(\Omega)\right)} \\
& +\int_{t_{01}}^{t}\left\|e^{\Delta(t-s)}\right\|_{0, \frac{1}{2}}\|f(u(s))\|_{L^{2}(\Omega)} d s
\end{aligned}
$$

pero) $\left\|e^{\Delta\left(t-t_{0}\right)}\right\|_{\frac{1}{2} \cdot \frac{1}{2}} \leq C e^{-\lambda_{1}\left(t-t_{0}\right)}$, que cstá acotado pral'a tiompo finito. $\left\|e^{\Delta(t-s)}\right\|_{0, \frac{1}{2}} \leq C(t-s)^{-\frac{1}{2}}$ y cultoncess

$$
\|u(t)\|_{\left.H_{0}^{\prime}(\Omega)\right)} \leq C_{T}+\int_{t_{0}}^{t} C(t-s)^{-\frac{1}{2}} \| f f\left(u(s) \|_{L^{2}(\Omega)} d s\right.
$$

Ahora bien.

$$
\|f(u(s))\|_{L^{2}(\Omega)} \leq C\|f(u(s))\|_{L^{\infty}(\Omega)} \leq C_{T}
$$

$\mathrm{y}$ por tanto

$$
\begin{aligned}
\int_{t_{0}}^{t} C(t-s)^{-\frac{1}{2}}\|f(u(s))\|_{L^{2}(\Omega)} d s & \leq C_{T} \int_{t_{1}}^{t}(t-s)^{-\frac{1}{2}} d s \\
& \leq C_{T} \int_{t_{1}}^{T}(T-s)^{-\frac{1}{2}} d s \leq C_{T}
\end{aligned}
$$


como la integral es finita. Luego,

$$
\|u(t)\|_{H_{0}^{1}(\Omega)} \leq C_{T}\left(T,\left\|u_{0}\right\|_{H_{0}^{1}(\Omega)}\right)
$$

con lo que tenemos la existencia de solución del problema en $\left[t_{0}, T\right]$ para todo $T>0$. Hemos obtenido que la solución es acotada, en intervalos finitos, con cotas dependientes de $\left\|u_{0}\right\| \in X^{\frac{1}{2}}$, y el intervalo $\left[t_{0}, T\right]$. Luego concluímos que la solución $\|u(t)\|_{H_{0}^{1}(\Omega)}$ es global en $H_{0}^{1}(\Omega) \cdot \square$

\section{Condición del tipo $f^{\prime}(s) \leq M$}

Teorema 7.1. Si $f$ verifica

$$
f^{\prime}(s) \leq M, \text { para todo } s \in \mathbb{R} \text {. }
$$

Entonces para todo $u_{0} \in L^{2}(\Omega)$ existe solución global del problema (5).

Demostración. Observamos que la condición (54) sobre $f$ implica en particular, que $f$ verifica

$$
s f(s) \leq M|s|^{2}+|f(0)||s|
$$

que es la condición (28) de la Sección§ 6 , con $C_{0}=-M$ y $C_{1}=|f(0)|$. Por tanto, si $u_{0} \in H_{0}^{1}(\Omega)$ : podemos aplicar el Teorema 6.1 y obtenemos existencia de solución global.

En efecto, si $u_{0} \in L^{2}(\Omega)$, sea $u_{0}^{n} \in H_{0}^{1}(\Omega)$ una sucesión de Cauchy en $L^{2}(\Omega)$ convergente a $u_{0}$. Sea $u^{n}(t)$ la solución global del problema (5) con dato inicial $u_{0}^{n}$, (sabemos que existe por la observación precedente). Restando las ecuaciones que verifican $u^{n}(t)$ y $u^{m}(t)$ y llamando $z(t)=u^{n}(t)-u^{m}(t)$, este satisface

$$
z^{\prime}(t)-\Delta z(t)=\left[f\left(u^{n}(t)\right)-f\left(u^{m}(t)\right)\right]
$$

Multiplicando, por $z(t)$ e integrando en $\Omega$ se obtiene,

$$
\frac{d}{d t}\|z(t)\|_{L^{2}(\Omega)}^{2}+\|\nabla z(t)\|_{L^{2}(\Omega)}^{2}=\int_{\Omega}\left[f\left(u^{n}(t)\right)-f\left(u^{m}(t)\right)\right] z(t) .
$$

Por el teorema del valor medio y la estimación sobre $f^{\prime}$; el segundo miembro podemos acotarlo por $M\|z(t)\|_{L^{2}(\Omega)}^{2}$ y se tiene

$$
\frac{d}{d t}\|z(t)\|_{L^{2}(\Omega)}^{2}+\|\nabla z(t)\|_{L^{2}(\Omega)}^{2} \leq M\|z(t)\|_{L^{2}(\Omega)}^{2}
$$

Si llamamos $y(t):=\|z(t)\|_{L^{2}(\Omega)}^{2}$ : entonces

$$
y^{\prime}(t) \leq M y(t)
$$

integrando en $(0, t)$ obtenemos

$$
y(t) \leq e^{M t} y(0) .
$$

Fijamos $0<t<\infty y$ la desigualdad anterior se traduce en

$$
\left\|u^{n}(t)-u^{m}(t)\right\|_{L^{2}(\Omega)}^{2} \leq e^{M t}\left\|u_{0}^{n}(t)-u_{0}^{m}(t)\right\|_{L^{2}(\Omega)}^{2}
$$

Tomando ahora supremo en $[0, t]$ : tenemos

$$
\sup _{0 \leq t \leq T}\left\|u^{n}(t)-u^{m}(t)\right\|_{L^{2}(\Omega)}^{2} \leq e^{M t}\left\|u_{0}^{n}(t)-u_{0}^{m}(t)\right\|_{L^{2}(\Omega)}^{2}
$$

y como la sucesión $\left\{u_{0}^{n}\right\}_{n \geq 1}$ es de Cauchy en $L^{2}(\Omega)$, tenemos que $\left\{u^{n}\right\}_{n \geq 1}$ es de Cauchy en $C\left([0, T] ; L^{2}(\Omega)\right)$ y por tanto, existe $u \in C\left([0, T] ; L^{2}(\Omega)\right)$ tal que $u^{n} \rightarrow u$ en tal espacio.

Integrando (58) en tiempo, obtenemos que

$$
\int_{0}^{T}\|\nabla z(t)\|_{L^{2}(\Omega)}^{2} \leq\|z(0)\|_{L^{2}(\Omega)}^{2}+M \int_{0}^{T}\|z(t)\|_{L^{2}(\Omega)}^{2} \leq C\left\|u_{0}^{n}-u_{0}^{n}\right\|_{L^{2}(\Omega)}
$$

lo que implica que $\left\{u^{n}\right\}_{n \geq 1}$ también es de Cauchy en $L^{2}\left((0, T) H_{0}^{1}(\Omega)\right)$.

Veamos ahora que $u(t)$ es solución del problema (5), es decir; que verifica la fórmula de variación de las constantes. Sabemos que para $t \geq r>0, u^{n}(t)$ viene dado en su forma integral por

$$
u^{n}(t)=e^{\Delta(t-r)} u^{n}(r)+\int_{r}^{t} e^{\Delta(t-s)} f\left(u^{n}(s)\right) d s
$$


Si bien, sabemos que $u^{n}(r)$ converge a $u(r)$ en $L^{2}(\Omega)$ cuando $n \rightarrow \infty$, basta probar que podemos pasar al límite en la integral.

Veamos si es posible encontrar una estimación de la forma

$$
\left\|u^{n}(t)\right\|_{L^{\infty}}(\Omega) \leq C_{T}, \text { para todo } 0<r \leq t \leq T \text {. }
$$

con $C_{T}$ independiente de $n$.

Por la ecuación (55) podemos como en la Sección $\S 6$, definir $w^{+}\left(t,\left|u_{0}^{n}\right|\right)$ de manera análoga y obtener

$$
\left|u_{n}(t)\right| \leq w^{+}\left(t,\left|u_{0}^{n}\right|\right)
$$

Utilizando como antes, la fórmula de variación de las constantes para $w^{+}$tenemos

$$
\left\|w^{+}\left(t,\left|u_{0}^{n}\right|\right)\right\|_{L^{\infty}(\Omega)} \leq\left\|e^{\left(\Delta-C_{0}\right) t} u_{0}^{n}\right\|_{L^{\infty}(\Omega)}+\int_{0}^{t}\left\|e^{\left(\Delta-C_{0}\right)(t-s)} C_{1}\right\|_{L^{\infty}(\Omega)} d s
$$

de manera análoga a lo realizado en la sección anterior obtenemos

$$
\left\|w^{+}\left(t,\left|u_{0}^{n}\right|\right)\right\|_{L^{\infty}(\Omega)} \leq C e^{D t} t^{\frac{-N}{1}}\left\|u_{0}^{n}\right\|_{L^{2}(\Omega)}+C_{T} \leq C_{T}
$$

para algún $D \in \mathbb{R}$, y $0<r \leq t \leq T$, puesto que $\left\{u_{0}^{n}\right\}_{n \geq 1}$ está acotada en $L^{2}(\Omega)$, y estamos en un intervalo acotado en tiempo de la forma $[r, T]$, con $r>0$, seguimos que

$$
\left\|w^{+}\left(t,\left|u_{0}^{n}\right|\right)\right\|_{L^{\infty}(\Omega)} \leq C_{T}
$$

por consiguiente

$$
\left\|u^{n}(t)\right\|_{L^{\infty}(\Omega)} \leq C_{T}
$$

con $C_{T}$ independiente de $n$. Como $\left\{u_{n}\right\}_{n \geq 1}$ converge a $u$ en $C\left([0, T] ; L^{2}(\Omega)\right)$ : podemos pasar al limite y obtenemos

$$
\|u(t)\|_{L^{\infty}(\Omega)} \leq C_{T}, \text { para todo } r \leq t \leq T .
$$

Luego podemos truncar $f$ a la altura $C_{T}$ y definir

$$
\tilde{f}(s)= \begin{cases}f\left(-C_{T}\right), & \text { si } s<-C_{T} \\ f(s), & \text { si }|s| \leq C_{T} \\ f\left(C_{T}\right), & \text { si } s>C_{T}\end{cases}
$$

que es una función globalmente Lipschitziana. Si sustituimos ahora en nuestro problema, el segundo miembro $f$ por $\tilde{f}$ tenemos un problema equivalente. Por tanto, podemos suponer que $f$ es globalmente Lipschitziana y por tanto

$$
\left|f\left(u^{n}(s)\right)-f(u(s))\right| \leq L\left|u^{n}(s)-u(s)\right|
$$

donde $L$ es la constante de Lipschitz de $f$.

Por consiguiente tenemos que, para todo s, $f\left(u^{n}(s)\right)-f(u(s)) \in L^{\infty}(\Omega) \subset L^{2}(\Omega)$. Por tanto

$$
\begin{aligned}
\| \int_{\tau}^{t} e^{\Delta(t-s)}\left(f\left(u^{n}(s)-f(u(s))\right) d s \|_{L^{2}(\Omega)}\right. & \leq \int_{\tau}^{t}\left\|e^{\Delta(t-s)}\right\|_{0,0} \|\left(f\left(u^{n}(s)-f(u(s))\right) \|_{L^{2}(\Omega)} d s\right. \\
& \leq L \int_{\tau}^{t} e^{-\lambda_{1}(t-s)}\left\|u^{n}(s)-u(s)\right\|_{L^{2}(\Omega)} d s \\
& \leq L \int_{\tau}^{t}\left\|u^{n}(s)-u(s)\right\|_{L^{2}(\Omega)} d s
\end{aligned}
$$

que tiende a cero cuando $n \rightarrow+\infty$, pues $u_{n}(t)$ converge a $u(t)$ uniformemente en $L^{2}(\Omega) \cdot \square$ 


\section{Conclusiones}

Dentro de un marco funcional de espacios de Sobolev, con condiciones de crecimiento en la nolinealidad hemos encontrado soluciones locales, del problema (5).

Revisamos algunos resultados de monotonía y positividad en un problema parabólico semilineal. Hacemos un estudio de soluciones globales de la ecuación (5) y presentamos nuestros resultados:

(1) Si imponemos una condición de estructura o de signo a la no lincalidad $f$ del tipo

$$
s f(s) \leq-C_{0}|s|^{2}+C_{1}|s|
$$

utilizando los resultados de positividad y monotonía y la fórmula de variación de las constantes obtenemos la existencia global de las soluciones.

(2) Si imponemos en la no linealidad una condición del tipo $f^{\prime}(s) \leq M$, obtenemos solución global.

(3) Obtenemos estimaciones para la solución de la ecuación (5) en espacios finos.

Primero veremos que podemos encontrar una estimación para $u^{n}(t)$ de la forma $\left\|u^{n}(t)\right\|_{L^{\infty}(\Omega)} \leq C_{T}$, para todo $0<r<t<T$, con $C_{T}$ independiente de $n$. En efecto, por la observación procedemos como en la sección anterior y definir $w^{+}\left(t,\left|u_{0}^{n}\right|\right)$ de manera análoga y obtenemos asıque se satisface

$$
\left|u_{n}(t)\right| \leq w^{+}\left(t,\left|u_{0}^{n}\right|\right)
$$

Utizando como antes, la fórmula de variación de las constantes para $w^{+}$tenemos

$$
\left\|w^{+}\left(t,\left|u_{0}^{n}\right|\right)\right\|_{L^{\infty}(\Omega)} \leq\left\|e^{\left(\Delta-C_{0} t\right) u_{0}^{n}}\right\|_{L^{\infty}(\Omega)}+\int_{0}^{t}\left\|e^{\left(\Delta-C_{0}\right)(t-s)} C_{1}\right\|_{L^{\infty}(\Omega)} d s
$$

de donde, de manera análoga a lo realizado en la sección anterior, por el Lema 3 VER $\left\|w^{+}\left(t,\left|u_{0}^{n}\right|\right)\right\|_{L^{\infty}(\Omega)} \leq$ $C e^{D t} t^{\frac{-N}{4}}\left\|u_{0}^{n}\right\|_{L^{2}(\Omega)}+C_{T} \leq C_{T}$ para cierto $D \in \mathbb{R}$ y $0<r \leq t \leq T$, ya que $\left\{u_{0}^{n}\right\}_{n \geq 1}$ está acotada en $L^{2}(\Omega)$ y estamos en un intervalo de tiempo de la forma $[r, T]$ con $r>0$.

Luego

$$
\left\|w^{+}\left(t,\left|u_{0}^{n}\right|\right)\right\|_{L^{\infty}(\Omega)} \leq C_{T}
$$

y por consiguiente

$$
\left.\| u^{n}(t)\right) \|_{L^{\infty}(\Omega)} \leq C_{T}
$$

con $C_{T}$ independiente de $n$. Como $u^{n}$ converge a $u$ en $C\left([0, T] ; L^{2}(\Omega)\right)$, podemos pasha al limite y tenemos

$$
\|u(t)\|_{L^{\infty}(\Omega)} \leq C_{T} \text {, para todo } r \leq t \leq T .
$$

Luego podemos truncar $f$ a la altura $C_{T}$ y definir de esta forma

$$
f(s)=\left\{\begin{array}{l}
f\left(-C_{T}\right) \text {; si } s<-C_{T} \\
f(s) \text { si }|s| \leq C_{T} \\
f\left(C_{T}\right) \text { si } s>C_{T}
\end{array}\right.
$$

que es una función globalmante lipschitziana. Si sustituimos en nuestro problema el segundo mienbro $f$ por $f$ tenemos un problema equivalente. Por consiguiente, podemos suponer que $f$ es globalinente lipschitziana y entonces

$$
\left|f\left(u^{n}(s)\right)-f(u(s))\right| \leq L\left|u^{n}(s)-u(s)\right|
$$

donde $L$ es la constante de Lipschitz de $f$. Ahora, para todo s, $f\left(u^{n}(s)-f(u(s)) \in L^{\infty}(\Omega) \subset L^{2}(\Omega)\right.$. Por tanto

$$
\begin{aligned}
\| \int_{r}^{t} e^{\Delta(t-s)}\left[f\left(u^{n}(s)-f(u(s))\right] d s \|_{L^{2}(\Omega)}\right. & \leq \int_{r}^{t}\left\|e^{\Delta(t-s)}\right\|_{0,0}\left\|f\left(u^{n}(s)\right)-f(u(s))\right\|_{L^{2}(\Omega)} d s \\
& \leq L \int_{r}^{t} e^{-\lambda_{1}(t-s)}\left\|u^{\prime 2}(s)-u(s)\right\|_{L^{2}(\Omega)} d s \\
& \leq L \int_{r}^{t}\left\|u^{n}(s)-u(s)\right\|_{L^{2}(\Omega)} d s
\end{aligned}
$$


que tiende a 0 cuando $n \rightarrow+\infty$ ya que $u_{n}$ converge a $u$ uniformemente en $L^{2}(\Omega)$. De manera similar a lo trabajado en (75) mpodemos tener más información tenieņdo en cuenta que $u^{n}(t)$ converge a $u(t)$ en $C^{0}\left([0, T] ; L^{2}(\Omega)\right)$.

En efecto, teníamos

$$
\begin{aligned}
\| \int_{r}^{t} e^{\Delta(t-s)}\left[f\left(u^{n}(s)-f(u(s))\right] d s \|_{X^{\alpha}}\right. & \leq \int_{r}^{t}\left\|e^{\Delta(t-s)}\right\|_{0,0}\left\|f\left(u^{n}(s)\right)-f(u(s))\right\|_{L^{2}(\Omega)} d s \\
& \leq C \int_{r}^{t}(t-s)^{-\alpha}\left\|u^{n}(s)-u(s)\right\|_{L^{2}(\Omega)} d s \\
& \leq C \sup _{r \leq s \leq T}\left\|u^{n}(s)-u(s)\right\|_{L^{2}(\Omega)} \int_{r}^{t}(t-s)^{-\alpha} d s
\end{aligned}
$$

donde la integral es finita para $\alpha<1$. Además para todo $r, t$ tales que $0<r<r_{0}<t<T$

$$
\left\|e^{\Delta(t-s)}\left(u^{n}(r)\right)-(u(s))\right\|_{\left.X^{*}\right)} \leq\left\|e^{\Delta(t-r)}\right\|_{0,0}\left\|u^{n}(r)-u(s)\right\|_{\left.L^{2}(\Omega)\right)} \leq C_{T}\left\|u^{n}-u\right\|_{C\left([r, T] ; L^{2}(\Omega)\right)} .
$$

Luego, para $t>\tau_{0}$,

$$
\left\|u^{n}(t)-u(t)\right\|_{X^{\infty}} \leq C_{T}\left\|u^{n}-u\right\|_{C^{\prime}[r, T] ; L^{2}(\Omega)}
$$

Por consiguiente, tomando supremo en $t \in\left[r_{0}, T\right]$ tenemos que $u^{n}$ converge a $u$ en $\left.C[r, T]: X^{\alpha}\right)$ parq todo $\alpha<1$ y todo $0<r_{0} \leq T<\infty$. En particular, para $\alpha=\frac{1}{2}$ tenemos la convergencia en $C\left([r, T] ; H_{0}^{1}(\Omega)\right)$ para todo $0<r<T$.

Suponiendo que $f(0)=0$ y procediendo de manera an"aloga, pero usando ahora la convergencia en $C\left([r, T] ; H_{0}^{1}(\Omega)\right)$ en vez de $C^{0}\left([0, T] ; L^{2}(\Omega)\right)$ obtenemos la convergencia en $C\left([r, T] ; X^{\beta}\right)$ para todo $\beta<\frac{3}{2}$. En particular, la convergencia se da para $\beta=1$, tenemos que $X^{1}=H^{2} \cap H_{0}^{1}$. luego, $u_{n} \rightarrow u$ en $C\left([r, T] ; H^{2}(\Omega) \cap H_{0}^{1}(\Omega)\right)$.

Hemos probado que para todo $0<r<t<\infty, u$ verifica

$$
u(t)=e^{\Delta(t-r)} u(r)+\int_{r}^{t} e^{\Delta(t-s)} f(u(s)) d s
$$

Finalmente veremos que podemos pasha al limite cuando $r$ tiende a 0 y habremos probado que $u$ es solución del problema (1).

Por una parte,

$$
e^{\Delta(t-r)} u(r)-e^{\Delta t} u(0)=e^{\Delta(t-r)}\left(u(r)-u_{0}\right)+\left(e^{\Delta(t-r)}-e^{\Delta t}\right) u_{0}
$$

pero

$$
\left\|e^{\Delta(t-r)}\left(u(r)-u_{0}\right)\right\|_{L^{2}(\Omega)} \leq C\left\|\left(u(r)-u_{0}\right)\right\|_{L^{2}(\Omega)} \rightarrow 0
$$

ckando $r \rightarrow 0$ ya que $u(r) \rightarrow u_{0}$ en $L^{2}(\Omega)$. Además

$$
\left(e^{\Delta(t-r)}-e^{\Delta}\right) u_{0}=e^{\Delta(t-r)}\left(u_{0}-e^{\Delta r} u_{0}\right)
$$

y tomando ahora norma en $L^{2}(\Omega)$

$$
\left\|\left(e^{\Delta(t-r)}-e^{\Delta t}\right) u_{0}\right\|_{L^{2}(\Omega)} \leq C\left\|u_{0}-e^{\Delta r} u_{0}\right\|_{L^{2}(\Omega)} \rightarrow 0
$$

Cuando $r \rightarrow o$ por ser $e^{\Delta t}$ un semigrupo $C^{0}$. Luego

$$
e^{\Delta(t-r)} u(r) \rightarrow e^{\Delta t} u_{0}
$$

cuando $r$ tiende a 0 , lo que implica, por (76), que la integral $\int_{r}^{t} e^{\Delta(t-s) f(u(s))} d s$ converge con lo que, por la definición de integral impropia;

$$
\int_{r}^{t} e^{\Delta(t-s) f(u(s))} d s \rightarrow \int_{0}^{t} e^{\Delta(t-s) f(u(s))} d s
$$

cuando $r \rightarrow 0 \cdot \square$ 


\section{Bibliografía}

[1] Brezis, H. (1984) Analisis funcional teoría y aplicaciones. Alianza Editorial.

[2] Carvalho, A.N., Cholewa J. W., Dlotko, T. (2001), Abstract Parabolic Problems In Ordered Banach spaces. Colloquium Mathematicum, Vol. 90, 1-17.

[3] Henry, D. (1981), Geometric Theory of Semilinear Parabolic Equations, Lecture Notes Math, Vol 840, Springer, Berlin.

[4] Triebel, H. (1995), Interpolation Theory Function Spaces Differential Operators. 2nd edition, Johann Ambrosius Barth Verlag -Heidelberg. Leipzig. 